\title{
Differential Induction of Symptoms in Arabidopsis by P6 of Cauliflower mosaic virus
}

\author{
Weichang Yu, ${ }^{1}$ Jane Murfett, ${ }^{2}$ and James E. Schoelz ${ }^{1}$ \\ ${ }^{1}$ Department of Plant Microbiology and Pathology, University of Missouri, Columbia, Missouri-65211; ${ }^{2}$ Department of \\ Biological Sciences, University of Missouri, Columbia, Missouri-65211
}

Submitted 1 July 2002. Accepted 12 September 2002.

\begin{abstract}
The gene VI protein (P6) of Cauliflower mosaic virus (CaMV) functions as a virulence factor in crucifers by eliciting chlorotic symptoms in infected plants. The ability to induce chlorosis has been associated previously with P6 through gene-swapping experiments between strains and through the development of transgenic plants that express P6. The primary role that has been identified for P6 in the CaMV infection cycle is to modify the host translation machinery to facilitate the translation of the polycistronic CaMV 35S RNA. This function for P6 has been designated as the translational transactivator (TAV) function. In the present study, we have characterized an unusual variant of P6, derived from CaMV strain D4, that does not induce chlorosis upon transformation into Arabidopsis thaliana. The level of D4 P6 produced in transgenic Arabidopsis line D4-2 was comparable to the amount found in transgenic plants homozygous for W260 and CM1841 P6, two versions of P6 that induce strong chlorotic symptoms and stunting in Arabidopsis. A complementation assay proved that P6 expressed in the D4-2 line was functional, as it could support the systemic infection of a CM1841 mutant that contained a lethal frame-shift mutation within gene VI. This complementation assay allowed us to separately assess the contribution of CM1841 gene VI to symptom development versus the contribution of other CM1841 genes. Furthermore, a previous study had shown that the TAV activity of D4 P6 was comparable to that of W260 P6. That comparative analysis of TAV function, coupled with the characterization of the D4-2 transgenic line in the present paper, indicates that the TAV function of P6 may play only a minor role in the development of chlorotic symptoms.
\end{abstract}

Additional keyword: virus symptom induction.

One of the most common types of symptoms associated with systemic infections of viruses is a mosaic (Hull 2002). Mosaic symptoms consist of chlorotic areas in a leaf, either associated with veins or in interveinal areas, interspersed with other areas of green tissue called "dark green islands." Recent evidence indicates that dark green islands might arise through posttranscriptional gene silencing of the viral infection in those tissues (Moore et al. 2001; Xie et al. 2001). Consequently, one effect of the virus infection on the plant may be to induce a generalized chlorosis through a disruption of the integrity of the chloroplasts or an impairment of chloroplast development (Hull 2002). The plant responds by

Corresponding author: J. E. Schoelz; E-mail: schoelzj@missouri.edu; Fax: (573) 882-0588. limiting the infection in portions of the leaf, and the outcome is a mosaic. In some cases, it has been possible to identify the viral gene responsible for the induction of chlorosis through exchanges of genes between infectious clones of a virus. Thus, it has been shown that the coat proteins of Tobacco mosaic virus and Cucumber mosaic virus are responsible for the production of chlorotic symptoms (Dawson et al. 1988; Shintaku et al. 1992).

Cauliflower mosaic virus (CaMV) gene VI has been shown to play a key role in the formation of chlorotic symptoms (Daubert et al. 1984; Stratford and Covey 1989). This virulence function was first associated with the gene VI protein (P6) through gene-swapping experiments. The role of gene VI in virulence was confirmed when it was transformed into several species of plants and, in most cases, the transgenic plants exhibited virus-like symptoms (Bálasz 1990; Baughman et al. 1988; Goldberg et al. 1991; Schoelz et al. 1991; Takahashi et al. 1989). One notable exception is that of gene VI of Figwort mosaic virus, which does not induce symptoms in Datura inoxia (Goldberg et al. 1991).

Arabidopsis is one plant that does develop virus-like symptoms upon transformation with CaMV gene VI (Cecchini et al. 1997; Zijlstra and Hohn 1992). Cecchini and coworkers transformed Arabidopsis with gene VI from CaMV strains CabbBJI, Bari-1, and Baji-31, and they found that all three versions of gene VI were capable of inducing virus-like symptoms. Of these three viruses, Bari-1 was considered the mildest, and some individuals transformed with this gene VI product did not induce any symptoms. However, the lack of symptoms was generally correlated with low levels of expression of P6. Plants that expressed a high level of Bari-1 P6 were mildly chlorotic and much smaller than the nontransgenic controls (Cecchini et al. 1997). Thus even in the case of this mild virus, gene VI induced virus-like symptoms.

In addition to its role in virulence, CaMV gene VI also functions as an avirulence factor in some Nicotiana species and some Arabidopsis ecotypes. In particular, P6 of CaMV strain W260 elicits a hypersensitive response (HR) in N. edwardsonii (Király et al. 1999; Palanichelvam et al. 2000), whereas P6 of strain CM1841 elicits a nonnecrotic resistance response in $N$. bigelovii and A. thaliana ecotype Tsu-0 (Agama et al. 2002; Wintermantel et al. 1993). Furthermore, the P6 protein has at least one well-defined role in the virus infection cycle as a translational transactivator (TAV) of CaMV gene expression (Bonneville et al. 1989; Gowda et al. 1989). In this capacity P6 is thought to interact with host ribosomes to allow for reinitiation of translation on the polycistronic CaMV 35S RNA (Park et al. 2001). Interestingly, recent evidence has shown that the TAV function of P6 is separate from its role as an elicitor of HR (Palanichelvam and Schoelz 2002). 
In this study, we sought to identify a natural variant of CaMV gene VI that would not induce any symptoms in Arabidopsis. We found one version of gene VI, derived from strain D4, that did not elicit symptoms when expressed as a transgene. The integrity of the D4 transgene was investigated through the development of a complementation assay designed to determine whether the D4 transgene could support the systemic infection of a CaMV frameshift mutant virus that was incapable of expressing gene VI. Since a previous study had shown that the TAV activity of D4 P6 was comparable to that of W260 P6 (Palanichelvam and Schoelz 2002), the present study indicates that the TAV function of P6 may play only a minor role in the development of chlorotic symptoms.

\section{RESULTS}

Characterization of transgenic Arabidopsis lines that express CaMV gene VI.

CaMV strains elicit a wide range of symptoms in Arabidopsis, from severe chlorosis and stunting to virtually no effect on the plant (Cecchini et al. 1998; Leisner and Howell 1992; Melcher 1989). To identify versions of CaMV gene VI that might condition differential effects on symptom induction, we inoculated Arabidopsis ecotype Col-0 with CaMV strains D4, W260, and CM1841. Earlier studies had shown that chimeric viruses that contained D4 gene VI in combination with CM1841 genes I to V induced 'symptomless' infections in tur-

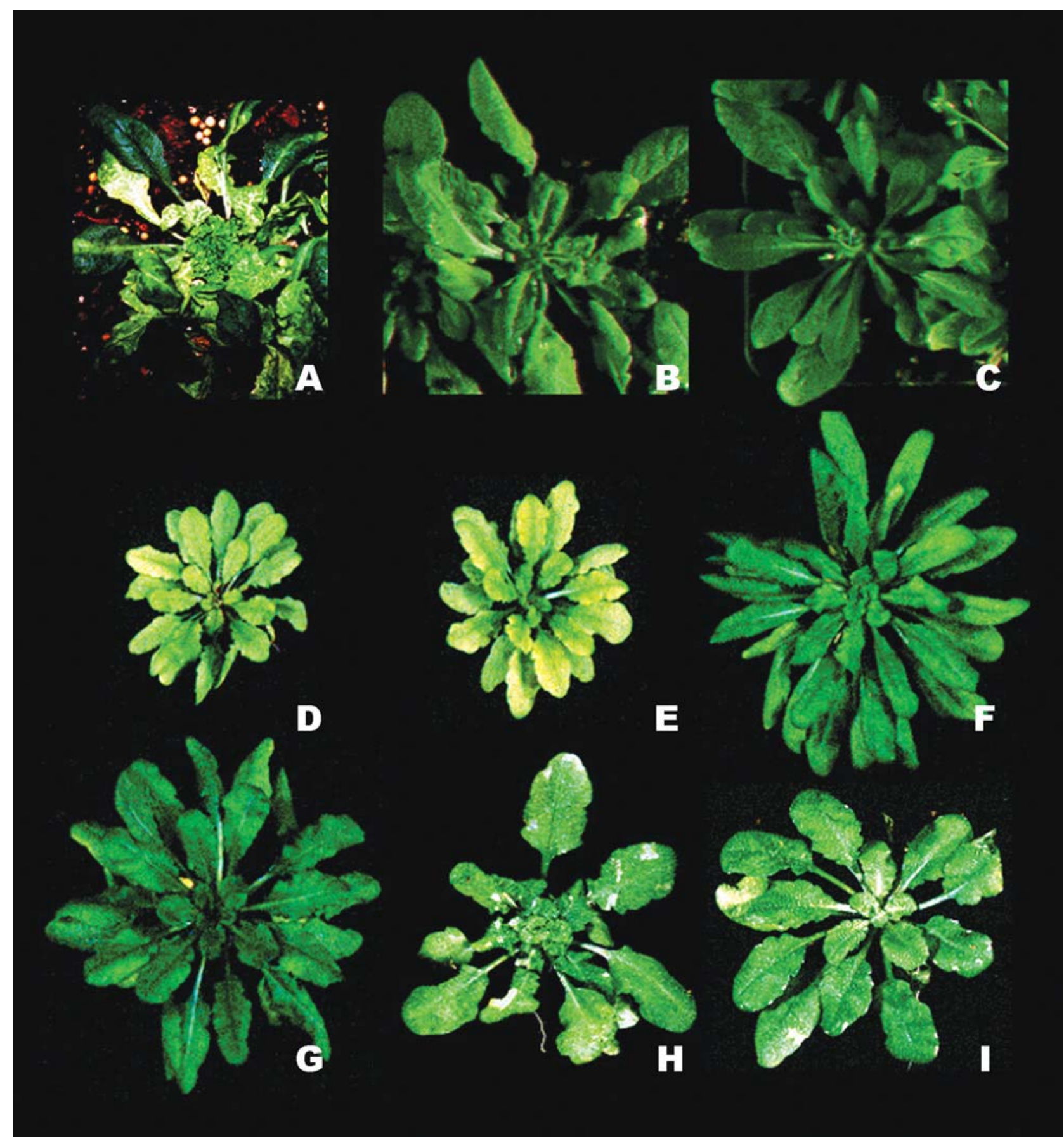

Fig. 1. Phenotypes of virus-infected Arabidopsis and transgenic Arabidopsis plants that express Cauliflower mosaic virus (CaMV) gene VI. Arabidopsis ecotype Col-0 is infected with A, CaMV strain CM1841 or B, CaMV strain D4. C, Uninfected plants. Virus-like symptoms induced in Arabidopsis plants transformed with gene VI from D, CM1841, E, W260, or F, D4. G, Nontransformed plants. Plants in D through G are from the same experiment. H, Transgenic line D4-2 infected with frameshift mutant J5215 and I, nontransformed Col-) control inoculated with J5215 were included in the same experiment. 
nips (Daubert et al. 1984; Schoelz and Wintermantel 1993). Consequently, D4 gene VI might be one candidate that might not elicit the virus-like symptoms associated with gene VI. We found that CM1841 and W260 induced typical CaMV symptoms in Arabidopsis. Older leaves exhibited a strong mosaic, whereas younger leaves were severely malformed (Fig. 1A). In addition, infected plants were severely stunted. In contrast, the D4 strain induced a mild mosaic and the leaves had some evidence of malformation (Fig. 1B).

To investigate whether transgenic expression of D4 gene VI would be incapable of eliciting symptoms in Arabidopsis, inflorescences were vacuum-infiltrated with Agrobacterium tumefaciens harboring gene VI constructs of D4, CM1841, or W260. Expression of each of these constructs was driven by the CaMV $35 \mathrm{~S}$ promoter present in the A. tumefaciens binary vector, pKYLX7 (Schardl et al. 1987). Seeds collected from the infiltrated plants were screened on Murashige-Skoog (MS) agar plates containing $50 \mu \mathrm{g}$ of kanamycin per ml to identify putative transformants. We isolated 8,32 , and 13 transgenic lines that expressed gene VI of D4, W260, and CM1841, respectively.

All of the lines transformed with gene VI of W260 and CM1841 exhibited typical virus-like symptoms associated with transgenic expression of P6 (Fig. 1D and E). These symptoms, which include stunting and generalized chlorosis, have been observed previously in Nicotiana species (Bálasz 1990; Baughman et al. 1988; Goldberg et al. 1991; Schoelz et al. 1991; Takahashi et al. 1989), as well as in Arabidopsis (Cecchini et al. 1997; Zijlstra and Hohn 1992). It is important to note that although these transgenic plants were stunted, the youngest leaves were not severely malformed as in the virusinfected plants (Fig. 1A). In contrast to results obtained with gene VI of CM1841 and W260, the lines transformed with gene VI of strain D4 were nearly symptomless (Fig. 1F). The only evidence of any effect of D4 P6 was that the leaves appeared to be slightly narrower than those of nontransformed Col-0 (Fig. 1F and 1G). This lack of symptoms indicated that P6 of D4 largely lacks the capacity to cause disease.

To investigate the inheritance of the D4 transgene, we collected seeds from three representatives of the $\mathrm{T}_{2}$ generation and plated them on solid media containing kanamycin $(50 \mu \mathrm{g} / \mathrm{ml})$. All three lines exhibited a 3:1 ratio for kanamycin resistance (Table 1), indicating that they each contained a transgenic locus. Significantly, none of the kanamycin-resistant plants from any of the three lines exhibited virus-like symptoms. Segregation of kanamycin resistance in $\mathrm{T}_{3}$ seedlings was used to identify a line, D4-2, that was homozygous for the D4-gene VI transgene. This line was used for subsequent experiments. Homozygous lines were also developed for the W260 and CM1841 gene VI transgenes.

The lack of virus-like symptoms in plants transformed with D4 gene VI might be a consequence of weak expression of the P6 protein. To investigate whether D4-2 plants expressed the D4 gene VI product, total proteins were isolated from plants, separated on a $12 \%$ protein gel, transferred to nitrocellulose, and probed with $\mathrm{P} 6$ antibodies, which specifically recognize a 10-amino acid sequence on the C-terminus of P6 (Schoelz et al. 1991). The Western blot revealed that D4-2 transgenic plants expressed a level of P6 comparable to that found in transgenic plants homozygous for W260 and CM1841 P6 and comparable to the level of P6 found in a virus-infected plant (Fig. 2). Although the sequence targeted by the antibodies is conserved in all three strains (Wintermantel et al. 1993), we cannot eliminate the possibility that subtle differences in P6 protein structure between strains might affect the detection of P6. In addition to the $62-\mathrm{kDa}$ P6 protein, we also detected proteins with sizes of approximately 35 and $25 \mathrm{kDa}$. These smaller proteins have been noted previously (Daubert and Routh 1990) and are thought to be breakdown products of P6. This study indicated that variations in the intensity of virus-like symptoms in transgenic plants can be caused by sequence variations within P6 as well as by differences in expression levels of P6, as shown previously by Cecchini and associates (1997).

To characterize the effect of CaMV P6 on the growth of Arabidopsis, we determined the dry weight of homozygous transgenic plants that expressed P6 of D4, W260, and CM1841 to nontransformed Col-0 (Fig. 3). The P6 of CM1841 and W260 severely affected the growth of Arabidopsis, as these plants accumulated approximately one fourth the mass of the nontransformed Col-0 plants. In contrast, the P6 protein of D4 had no significant effect on the mass of Arabidopsis plants.

\section{Transgenic D4-2 plants can complement a CaMV mutant that contains a lethal frame-shift within gene VI.}

Although the amount of D4 P6 produced in transgenic plants was comparable to that found in CaMV-infected plants, there was still a possibility that the 'symptomless' phenotype might be due to a defect in the D4 transgene. To determine whether the D4 P6 was functioning properly, we developed a complementation assay with the D4-2 transgenic line and a form of CaMV that contained a lethal mutation within gene VI. An 11bp deletion was introduced by polymerase chain reaction (PCR) into gene VI of strain CM1841 (Fig. 4A, nucleotides $5,810$ to 5,820$)$. Since CM1841 gene VI begins at nucleotide position 5,774 and ends at nucleotide 7,336 (Gardner et al. 1981), this deletion would result in a frameshift mutation that abolishes most of the protein product. The deletion in gene VI was then introduced into a partially redundant infectious clone that could be agroinoculated into plants (Grimsley et al. 1986). If the D4-2 transgenic line complemented this mutant virus, it would show that D4 P6 is fully functional.

The frameshift mutant virus, designated JS215, was unable to infect nontransformed Arabidopsis upon agroinoculation (0 of 40 inoculated), and it also failed to infect turnips (data not shown). In contrast, it could efficiently infect the D4-2 transgenic Arabidopsis plants. Transgenic plants (39 out of 40) exhibited very mild symptoms by 22 days postinfection (dpi). Significantly, these plants did develop a mild malformation in the youngest leaves (Fig. 1H and I). This is in agreement with other studies that have indicated that portions of the viral genome other than gene VI contribute to leaf malformation and stunting (Stratford and Covey 1989).

Several reports show that CaMV is able to recombine with a gene VI transgene (Gal et al. 1992; Schoelz and Wintermantel

Table 1. Segregation of D4 gene VI in transgenic Arabidopsis plants

\begin{tabular}{llll}
\hline Transgenic $\mathbf{T}_{\mathbf{1}}$ plant & $\mathbf{K m}^{\mathbf{r}} / \mathbf{K m}^{\mathbf{s}}$ in $\mathbf{T}_{\mathbf{2}} \mathbf{s e e d s}^{\mathbf{a}}$ & Symptoms/symptomless $^{\mathbf{b}}$ & P6 expression $^{\mathbf{c}}$ \\
\hline D4 \#1 & $76: 24$ & $0: 100$ & Yes \\
D4 \#2 & $75: 25$ & $0: 100$ & Yes \\
D4 \#3 & $78: 22$ & $0: 100$ & Yes \\
\hline
\end{tabular}

${ }^{\mathrm{a}}$ Ratios determined by germinating seed on agar supplemented with kanamycin $(50 \mu \mathrm{g} / \mathrm{ml}) . \mathrm{Km}^{\mathrm{r}}=$ resistant, $\mathrm{Km}^{\mathrm{s}}=$ sensitive.

${ }^{\mathrm{b}} \mathrm{T}_{2}$ plants with or without symptoms as determined by visual observation for virus-like symptoms.

${ }^{\mathrm{c}} \mathrm{T}_{2}$ and $\mathrm{T}_{3}$ lines positive for P6 expression as determined by Western blot with P6 antiserum. 
1993); consequently, it was important to determine whether the infections that developed in the D4-2 plants were due to complementation, recombination with the transgene, or restoration of gene VI function due to a second-site mutation. To analyze the virus present in the transgenic plants, we isolated $\mathrm{CaMV}$ virions from nine infected D4-2 plants and then degraded chromosomal DNA with DNAse I. Viral DNA was protected from the DNAse I treatment by the capsid shell (Gardner and Shepherd 1980) and was subsequently released from the virion by proteinase $\mathrm{K}$ digestion of the preparation. The source of gene VI could be identified due to polymorphisms in EcoRI sites within gene VI (Fig. 4A). A band of 397 bp would be indicative of recombination with the transgene sequences, whereas a DNA band of approximately 459 bp would be indicative of complementation (Schoelz and Wintermantel 1993). Each of the viral DNA samples was digested with EcoRI, and the DNA fragments separated on a $2.0 \%$ agarose gel. To visualize the DNA bands, they were transferred to Genescreen and probed with a ${ }^{32} \mathrm{P}$-labeled CaMV DNA probe. In eight of the nine samples we examined, there was no evidence of recombination. A representative isolate is shown in Figure 4B. In the remaining sample, either the viral DNA was lost during the purification process, or that plant had not been infected with JS215 (data not shown). Our results suggested that the JS215 virus was complemented for infection rather than undergoing recombination with the transgene.

To complete the characterization of the viruses responsible for infection of the D4-2 plants, we developed PCR primers to amplify the segment of gene VI that flanked the 11-bp deletion (Fig. 4A). In this experiment, $10 \mathrm{JS} 215$-inoculated plants were pooled, and then, virion DNA was isolated. Figure $4 \mathrm{C}$ shows the PCR products that were amplified from the virion DNA purified from transgenic plants and that were cleaved with EcoRI. The DNA band amplified from the JS215-inoculated plants (Fig. 4C, lane 5) matched the band amplified from purified JS215 plasmid DNA. It was clearly larger than the 278-bp product expected for the D4 transgene (Fig. 4C, lane 2). No CaMV sequences were amplified from uninfected transgenic Arabidopsis plants, demonstrating that the transgene had been degraded by DNAse I during the virion purification procedure

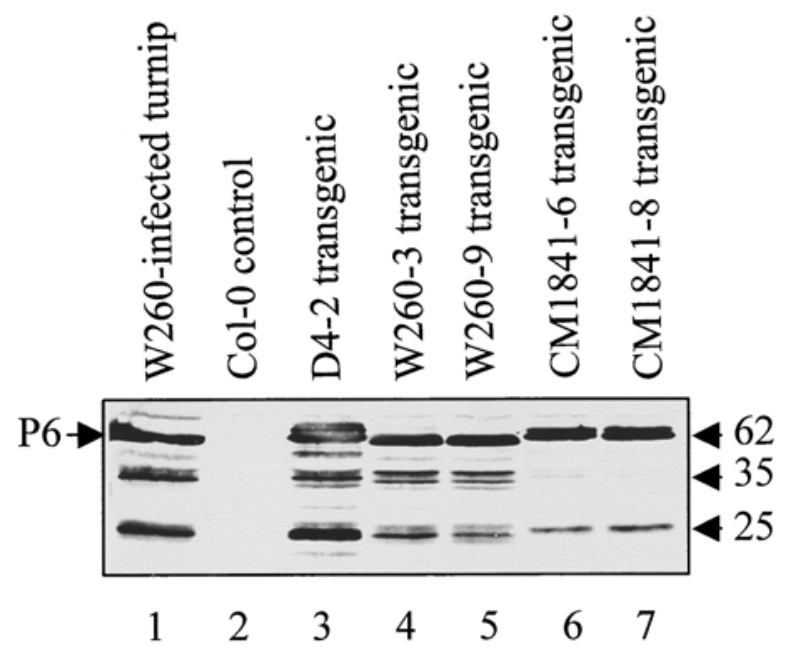

Fig. 2. Expression of gene VI protein (P6) in selected transgenic Arabidopsis lines. Equivalent amounts of protein from individual plants were separated by $12 \%$ sodium dodecyl sulfate-polyacrylamide gel electrophoresis, were transferred to a nitrocellulose membrane, and were probed with antiserum to Cauliflower mosaic virus $\mathrm{P} 6$. The arrow at the left indicates the position of P6; the arrows at the right indicate the fullsize band with a mass of $62 \mathrm{kDa}$, as well as the two breakdown products of $35 \mathrm{kDa}$ and $25 \mathrm{kDa}$.
(Fig. 4C, lane 6). Furthermore, no CaMV DNA was detected in upper leaves of nontransformed Col-0 inoculated with JS215 (Fig. 4C, lane 7). Nucleotide sequencing of the viral band recovered from JS215-inoculated plants confirmed that the viral DNA that we amplified had retained the 11-bp deletion. Together, the Southern blot and PCR analysis clearly indicated that the CaMV infections in the inoculated D4-2 plants were not the consequence of recombination between JS215 and the transgene.

To determine if a second-site mutation beyond the portion of gene VI that was amplified and sequenced had restored the function of gene VI, the virus responsible for JS215 infections in D4-2 plants was passaged to nontransformed Col-0 and to a new set of D4-2 plants. By $22 \mathrm{dpi}$, all four of the D4-2 plants had developed symptoms, whereas none of the four nontransformed Col-0 plants appeared to have become infected. The nontransformed Col-0 remained symptomless up to $34 \mathrm{dpi}$, and at that point, we attempted to purify virion DNA from both the transgenic and nontransformed plants. As in the analysis of the initially infected plants, the virion DNA preparations were treated with DNAse I to degrade the transgene DNA. Viral DNA sequences could be amplified by PCR from the D4-2 plants that had been inoculated with the JS215 virions, but CaMV sequences were not amplified from the nontransformed Col-0 plants (data not shown). This experiment demonstrated that the JS215 virus was stably maintained after a single passage, and that no compensating mutations had occurred within the viral copy of gene VI to restore its function.

To characterize the efficacy of complementation, we used an enzyme-linked immunosorbent assay (ELISA) to compare the titers of virion antigen in D4-2 transgenic plants that had been inoculated with wild-type W260 and JS215. The level of viral antigen in the JS215-inoculated plants was $72 \% \pm 7 \%(n=5)$ of the titer found in W260-inoculated plants. Thus, the D4-2 transgenic line could support a level of infection that was nearly comparable to that of a plant infected with a wild-type CaMV strain.

\section{DISCUSSION}

We have shown that transgenic plants that express gene VI of CaMV strain D4 are nearly symptomless, and a complementation assay proved that they do produce a functional copy of P6. The symptomless D4-2 line will be valuable for understanding how CaMV causes disease in its hosts, and it will also provide some insight into the factors that govern recombination of viruses with viral transgenes versus complementation of defective viruses by viral transgenes. In addition, the complementation experiments have implications for the expression strategy of CaMV.

\section{Dissection of symptom development in plants transformed with CaMV gene VI.}

Gene VI of Cauliflower mosaic virus has been transformed into several species of plants and, in most cases, the transgenic plants have exhibited virus-like symptoms (Bálasz 1990; Baughman et al. 1988; Goldberg et al. 1991; Schoelz et al. 1991; Takahashi et al. 1989). In the present study, we found that a gene VI transgene derived from CaMV strain D4 had very little impact on the growth of Arabidopsis. The only phenotypic difference between the D4 transgenic plants and nontransformed plants was that the leaves of the D4-2 transgenic plants appeared to be narrower than the nontransformed plants. In contrast, both the W260 and CM1841 transgenes elicited virus-like symptoms characterized by leaf chlorosis and stunting. Significantly, the level of D4 P6 was equivalent to the amount found in virus-infected plants. Furthermore, this D4 gene con- 
struct had been shown to have full TAV function (Palanichelvam and Schoelz 2002), an indication that the TAV function of P6 may play only a minor role, or perhaps no role, in the development of chlorotic symptoms. The lack of symptoms induced by the D4 gene VI transgene is consistent with how this version of P6 acts in virus-infected plants. In particular, a chimeric virus composed of D4 gene VI combined with genes I to V of CM1841 conditioned symptomless infections in turnips (Daubert et al. 1984; Schoelz and Wintermantel 1993).

The complementation assay that we developed allowed us to separately assess the role of CM1841 gene VI in symptom development in Arabidopsis and, conversely, to assess the role of CM1841 genes I to V in the absence of gene VI. The CM1841 gene VI transgene induced generalized chlorosis and severely affected plant growth, but leaves lacked the severe malformation exhibited by plants infected with the CM1841 virus. This can be contrasted with the symptoms that developed in the D42 plants infected with JS215, a derivative of CM1841 that could not produce its own P6 protein. These plants exhibited the leaf malformation characteristic of CM1841 virus infections, but only a very mild chlorosis. Thus, the 'stunting' phenotype associated with CM1841 virus infections may be further divided into factors that induce chlorosis and reduce plant growth (gene VI) and factors that induce leaf malformation (genes I to V). This distinction may provide a greater refinement of the map of CaMV symptom determinants (Stratford and Covey 1989; Hull, 2002).

Interestingly, there is evidence that the effect of the P6 transgene on Arabidopsis gene expression is similar to that of virus infections. Geri and coworkers (1999) used differential display techniques to compare the changes in gene expression that occur in transgenic plants with those that occur in virus infections. Although there were some differences in the expression patterns of the two types of plants, there were also many similarities. Geri and associates (1999) concluded that transgenic plants that express CaMV P6 could serve as a model system to investigate symptom development. It is true that other CaMV genes also contribute to symptom formation, but that specific symptom characteristics could be attributed to gene VI.

\section{Complementation vs. recombination with the D4 gene VI transgene.}

Several reports show that CaMV is able to recombine with a gene VI transgene (Gal et al. 1992; Király et al. 1998; Schoelz and Wintermantel 1993; Wintermantel and Schoelz 1996). The implication of these studies is that a recombinant between the mutant virus and transgene would be the predominant, or even the only, product. However, the predominance of recombinant viruses over defective viruses is not inevitable. Before it had been proven that CaMV could recombine with CaMV transgenes, an earlier study showed that transgenic Nicotiana bigelovii that expressed D4 P6 would complement a chimeric CaMV isolate that contained a host-specific defect within gene VI (Schoelz et al. 1991). In this case, the chimeric virus H31 could produce a functional P6 protein that allowed that virus to infect turnips systemically, but a defect that mapped to gene VI prevented $\mathrm{H} 31$ from infecting nontransformed $N$. bigelovii.

The experiments with the chimeric virus H31 can be contrasted with those with JS215, which contained a frameshift mutation within gene VI that would prevent it from producing any P6 protein. Since the infections we observed in Arabidopsis might be explained by either recombination or complementation, it was important to investigate the genetic composition of the viruses that were found in upper, noninoculated leaves. With our present work, we have shown that not only are hostspecific defects complemented, transgenic plants may also complement CaMV isolates that lack the capacity to express P6.
The factors that determine whether CaMV will be complemented by a transgenic plant or will undergo recombination with the transgene are likely related to either the nature of the defect within the inoculated virus or to the structure of the transgene. For example, a CaMV isolate that lacks essential cis-acting elements within a gene will likely undergo recombination with the transgene to restore those essential sequences. In theory, however, it should be possible to complement a virus in which large portions of gene VI have been eliminated from the virus. This may allow the development of a two-component vector system for Arabidopsis based on expression of P6 from a transgene and expression of a foreign gene from the 19S promoter present in the virus genome.

Transgene structure may also influence the outcome of complementation or recombination. In our transgene construct, we used the $r b c S$ termination signal, whereas previous studies had relied on the termination signals associated with the CaMV 19S RNA (Gal et al. 1992; Schoelz and Wintermantel 1993). The use of the 19S termination signal might facilitate recombination between the 35S RNA and the transgene mRNA during reverse transcription of the viral genome. The viral reverse transcriptase must make a template switch at that point during replication of the virus, and the presence of the natural end of the 19S transcript would be an ideal template for recombination (Gal et al. 1992; Schoelz and Wintermantel 1993). In contrast, the $3^{\prime}$ end of the transcript produced in our transgenic Arabidopsis would reduce the number of sequences that are shared between the transgene and the 35S RNA. The reverse transcriptase might prefer to switch to the $3^{\prime}$ end of the $35 \mathrm{~S}$ RNA rather than the heterologous $3^{\prime}$ end present on the transgene. Consequently, recombinant viruses may form less frequently when a heterologous $3^{\prime}$ end is used and have little chance to become established in the plant. A similar effect has been noted previously in studies on recombination between RNA viruses and viral transgenes (Greene and Allison 1996).

\section{CaMV P6 can be supplied in trans to support a systemic infection.}

In wild-type virus infections, $\mathrm{P} 6$ is expressed as a monocistronic transcript from the $19 \mathrm{~S}$ promoter (Odell and Howell 1980). The $19 \mathrm{~S}$ promoter is considered to be much weaker than

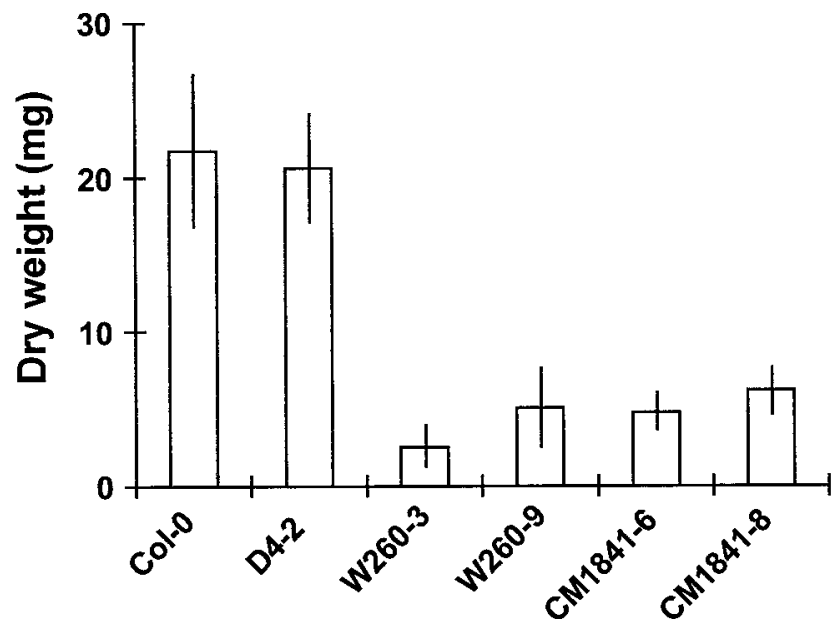

\section{Arabidopsis lines}

Fig. 3. Influence of gene VI protein (P6) expression on the growth of Arabidopsis. Plants (20) from each of the homozygous Arabidopsis lines were grown in a growth chamber at $22^{\circ} \mathrm{C}(14 \mathrm{~h}$ light and $10 \mathrm{~h}$ dark) for 45 days. Individual plants were harvested, were dried in an incubator, and then were weighed. 
the 35S promoter (Driesen et al. 1993; Lawton et al. 1987), and it has been considered that the level of P6 produced from the 19S RNA might be insufficient to support a virus infection. The discovery that P6 can transactivate its own expression from the 35S RNA (Driesen et al. 1993; Scholthof et al. 1992) provided one hypothesis for how large amounts of P6 might be produced. As P6 accumulates in the cell, it would transactivate its own translation from the $35 \mathrm{~S}$ RNA and, late in infection, this might be the preferred manner for expression of gene VI.

In this study, we tested whether there was an absolute requirement for expression of P6 from the 35S RNA. A previous paper had found that P6 could be supplied in trans in protoplasts (Kobayashi et al. 1998), but there are significant hurdles for viruses to overcome in moving from a single cell to other portions of the plant. We were able to extend this observation by showing that sufficient levels of P6 could be supplied in trans to sustain a systemic infection. The key to CaMV infections may be the attainment of a threshold level of P6 in the cell, which might occur in cis in nature, but can be accomplished in trans by genetic manipulation. If a certain concentration of P6 is required, then it would be interesting to see if virus infections could be maintained when P6 is supplied by a weaker promoter than the $35 \mathrm{~S}$ promoter.

\section{MATERIALS AND METHODS}

Transformation of Arabidopsis.

The W260 and D4 gene VI constructs used for transformation of Arabidopsis have been described previously (Palanichelvam et
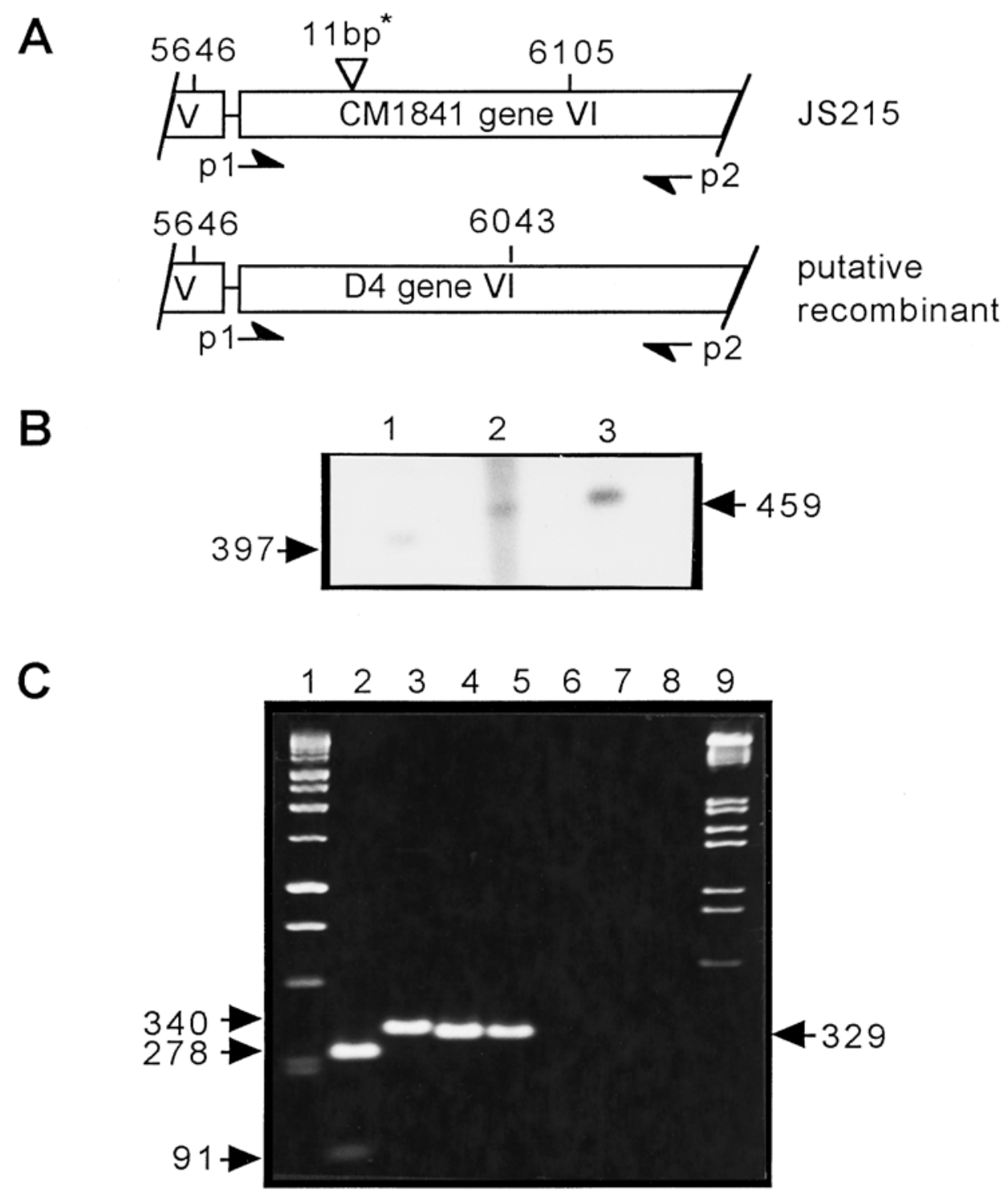

Fig. 4. Analysis of Arabidopsis plants agroinoculated with JS215, a partially redundant clone of strain CM1841 that contains a lethal frameshift mutation within gene VI. A, Structure of gene VI from JS215 (top) and the putative virus that would form upon recombination with the D4 gene VI transgene (bottom). JS215 contains an 11-bp deletion from nucleotides 5,810 to 5,820. Both viruses have an EcoRI site at nucleotide position 5,646. JS215 has a second EcoRI site at position 6,105, whereas the putative recombinant would acquire an EcoRI site at position 6,043 from the D4 gene VI transgene (Schoelz and Wintermantel 1993). Positions of two polymerase chain reaction (PCR) primers, p1 and p2, with 5' ends at 5,774 and 6,134, respectively, are shown. B, Southern blot analysis of cloned viral DNAs and JS215 DNA isolated from an agroinfected D4-2 plant. DNA samples were cleaved with EcoRI and probed with CM1841 DNA. Samples were derived from cloned D4 viral DNA (lane 1), JS215 viral DNA isolated from a D4-2 plant (lane 2), and CM1841 cloned DNA (lane 3). C, PCR analysis of viral DNAs. The PCR products were digested with EcoRI and analyzed on 2\% agarose stained with ethidium bromide. Samples were derived from D4 cloned DNA (lane 2), CM1841 cloned DNA (lane 3), JS215 cloned DNA (lane 4), JS215 DNA isolated from a D4-2 plant (lane 5), an uninoculated D4-2 transgenic plant (lane 6), nontransformed Arabidopsis Col-0 agroinoculated with JS215 (lane 7), and an uninoculated Col-0 plant (lane 8). A 1-kb marker DNA standard is in lane 1, and a lambda DNA standard cleaved with EcoRI and HindIII is in lane 9. 
al. 2000). These gene VI constructs were made in the Agrobacterium vector, pKYLX7 (Schardl et al. 1987). The 35S promoter is used for expression of transgenes in pKYLX7, and the terminator sequence is derived from the small subunit of ribulose-1,5-bisphosphate carboxylase gene $(r b c S)$. To make the CM1841 gene VI construct, the gene VI coding region was amplified by PCR from plasmid pS10 (Gardner et al. 1981), a plasmid that contains the entire CM1841 genome. Oligonucleotide primers were designed in a manner such that an XhoI site was added immediately upstream of the gene VI initiation codon, and a KpnI site was placed immediately downstream of gene VI termination codon. The amplified CM1841 gene VI sequence was subsequently cloned into the XhoI -KpnI sites of pKYLX7. All constructs were mobilized into A. tumefaciens GV2260 by triparental mating (Ditta et al. 1980).

A. thaliana ecotype Columbia was grown in a growth chamber at $22^{\circ} \mathrm{C}$ under continuous light conditions and was transformed by the vacuum infiltration method (Bechtold et al. 1993). Infiltrated plants were returned to the growth chamber set at the same conditions as before. Seeds from infiltrated plants were collected and transformants selected on MS medium (Murashige and Skoog 1962) supplemented with $50 \mu \mathrm{g}$ of kanamycin per ml. Kanamycin-resistant plants were transferred to the soil and were selfed for segregation analysis.

\section{Western analysis for expression of $\mathrm{P6}$ protein.}

The Western blot for CaMV P6 was conducted according to Király and associates (1999). Leaves were homogenized 1:3 (wt/vol) in loading buffer, were boiled for $5 \mathrm{~min}$, and the homogenate was centrifuged for $30 \mathrm{~s}$ at $12,000 \times g$. The supernatant was diluted 10 -fold with loading buffer $(0.125 \mathrm{M}$ Tris [pH 6.8], 4\% [wt/vol] sodium dodecyl sulfate, $20 \%$ [vol/vol] glycerol, and $10 \%$ [vol/vol] 2-mercaptoethanol), and proteins were separated on a $12 \%$ acrylamide gel (Laemmli, 1970). Proteins were transferred to nitrocellulose membranes for $1 \mathrm{~h}$ at 400 milliamperes (constant current) and were blocked overnight in blocking buffer (phosphate buffered saline [PBS, $\mathrm{pH}$ 7.4], 5\% [wt/vol] nonfat dry milk, and $1 \%$ [wt/vol] bovine serum albumin). Membranes were incubated at room temperature for 1.5 h with 1:500 dilution of anti-CaMV P6 antiserum (in PBS-Tween buffer [pH 7.4] containing $2.0 \mathrm{~g}$ BSA per 1 as the blocking agent), followed by an incubation for $1.5 \mathrm{~h}$ at room temperature with a 1:7,500 dilution of alkaline phosphatase-labeled goat-anti-rabbit IgG (Fisher Scientific, St. Louis). The antiserum to CaMV P6 was made previously against a peptide that corresponds to the C-terminal 10 amino acids of P6 (Schoelz et al. 1991). Between each step the membranes were washed with PBS (pH 7.4) with $0.05 \%$ Tween-20. Protein bands were visualized by addition of $10 \mathrm{ml}$ of alkaline phosphatase color developer (100 mM Tris [pH 9.1], $100 \mathrm{mM}$ $\mathrm{NaCl}, 5 \mathrm{mM} \mathrm{MgCl} 2$, and $0.4 \mathrm{mM}$ nitro blue tetrazolium chloride, $0.4 \mathrm{mM}$ 5-bromo-4-chloro-3-indolyl phosphate D-toluidine salt), which was allowed to react for 10 to $20 \mathrm{~min}$.

\section{Construction of the CaMV frame-shift mutant, JS215, and inoculation of plants.}

The CaMV clone used for agroinoculation, JS215 (Fig. 4), was made by deleting $11 \mathrm{bp}$ (from 5,810 to 5,820) of the gene VI region of CM1841 virus. The deletion was introduced by recombinant PCR (Higuchi 1990) into a SalI (nucleotide 4,833) to SacI (nucleotide 5,822) DNA segment derived from CaMV strain CM1841. The deletion was confirmed by sequencing the cloned SalI-SacI DNA segment at the DNA core facility at the University of Missouri. This SalI to SacI DNA segment was then exchanged with the corresponding wild-type segment of p306 to form JS215. Plasmid p306 is a partially re- dundant infectious clone of CaMV strain CM1841 inserted into the T-DNA border sequences of the disarmed Ti plasmid vector, pOCA28 (Király et al. 1998). The JS215 construct was mobilized into A. tumefaciens GV2260 by triparental mating. To initiate infections with JS215, Arabidopsis was grown in a growth chamber set at $22^{\circ} \mathrm{C}$ under short daylight conditions (10 h light and $14 \mathrm{~h}$ dark). Three-week-old plants were agroinoculated as described by Király and associates (1998). To initiate infections with CaMV virions, virions were partially purified according to Schoelz and associates (1986) and were gently rubbed on the young leaves of Arabidopsis plants. The ELISA to assess CaMV virion concentrations has been described previously (Anderson et al. 1991).

\section{Analysis of viral DNA from infected Arabidopsis.}

Viral DNA was purified as described by Gardner and Shepherd (1980). In this procedure, chromosomal DNA is degraded by DNAse I treatment, whereas encapsidated viral DNA is protected by the protein coat. Virions are subsequently treated with proteinase K to release viral DNA. For the Southern blot, viral DNA was cleaved with EcoRI, separated on a $2 \%$ agarose gel, transferred to a nylon membrane, and was probed with a ${ }^{32} \mathrm{P}$-labeled DNA probe as described in Maniatis and associates (1982). The probe consisted of full-length CM1841 DNA cloned into the plasmid vector pBR322.

For PCR, 21-mer primers were selected that would amplify a portion of gene VI from nucleotides 5,774 to 6,134. DNA segments amplified by PCR were digested with EcoRI and were analyzed on a $2 \%$ agarose gel stained with ethidium bromide. To clone the PCR fragment, an XhoI site was included on the forward primer such that, after amplification, an XhoI-EcoRI DNA fragment could be cloned into the plasmid pGEM7Zf(-) (Promega, Madison, WI, U.S.A.). The cloned PCR fragment was sequenced at the DNA core facility to investigate whether the 11-bp deletion within gene VI of JS215 had been retained.

\section{ACKNOWLEDGMENTS}

The authors thank A. Cole and K. Palanichelvam for technical assistance, J. Cawly for comments on the manuscript, and D. Pinkerton for assistance with the photographs. This research was supported by a grant from the Food for the 21 st Century program at the University of Missouri.

\section{LITERATURE CITED}

Agama, K., Beach, S., Schoelz, J., and Leisner, S. M. 2002. The 5' third of Cauliflower mosaic virus gene VI conditions resistance breakage in Arabidopsis Ecotype Tsu-0. Phytopathology 92:190-196.

Anderson, E. J., Qiu, S. G., and Schoelz, J. E. 1991. Genetic analysis of disease severity and virus concentration determinants in cauliflower mosaic virus. Virology 181:647-655.

Bálasz, E. 1990. Disease symptoms in transgenic tobacco induced by integrated gene VI of cauliflower mosaic virus. Virus Genes 3:205211.

Baughman, G. A., Jacobs, J. D., and Howell, S. H. 1988. Cauliflower mosaic virus gene VI produces a symptomatic phenotype in transgenic tobacco plants. Proc. Natl. Acad. Sci. U.S.A. 85:733-837.

Bechtold, N., Ellis, J., and Pelletier, G. 1993. In planta Agrobacterium mediated gene transfer by infiltration of adult Arabidopsis thaliana plants. Sciences de la vie 316:1194-1199.

Bonneville, J.-M., Sanfaçon, H., Fütterer, J., and Hohn, T. 1989. Posttranscriptional transactivation in cauliflower mosaic virus. Cell 59:1135-1143.

Cecchini, E., Gong, Z., Geri, C., Covey, S. N., and Milner, J. J. 1997. Transgenic Arabidopsis lines expressing gene VI protein from cauliflower mosaic virus variants exhibit a range of symptom-like phenotypes and accumulate inclusion bodies. Mol. Plant-Microbe Interact. 10:1094-101.

Cecchini, E., Al-Kaff, N. S., Bannister, A., Giannakou, M. E., McCallum, D. G., Maule, A. J., Milner, J. J., and Covey, S. N. 1998. Pathogenic interactions between variants of cauliflower mosaic virus and Arabi- 
dopsis thaliana. J. Exper. Bot. 49:731-737.

Daubert, S. D., and Routh, G. 1990. Point mutations in cauliflower mosaic virus gene VI confer host-specific symptom changes. Mol. PlantMicrobe Interact. 3:341-345.

Daubert, S. D., Schoelz, J. E., Debao, L., and Shepherd, R. J., 1984. Expression of disease symptoms in cauliflower mosaic virus genomic hybrids. J. Mol. Appl. Genet. 2:537-547.

Dawson, W. O., Bubrick, P., and Grantham, G. L. 1988. Modification of the tobacco mosaic virus coat protein gene affecting replication, movement and symptomatology. Phytopathology 78:783-789.

Ditta, G., Stanfield, S., Corbin, D., and Helinski, D. R. 1980. Broad host range DNA cloning system for gram-negative bacteria: Construction of a gene bank of Rhizobium meliloti. Proc. Natl. Acad. Sci. U.S.A. 77:7347-7351.

Driesen, M., Benito-Moeno, R.-M., Hohn, T., and Fütterer J. 1993. Transcription from the CaMV 19S promoter. Virology 195:203-210.

Gal, S., Pisan, B., Hohn, T., Grimsley, N., and Hohn, B. 1992. Agroinfection of transgenic plants leads to viable cauliflower mosaic virus by intermolecular recombination. Virology 187:525-533.

Gardner, R. C., and Shepherd, R. J. 1980. A procedure for rapid isolation and analysis of cauliflower mosaic virus DNA. Virology 106:159-161.

Gardner, R. C., Howarth, A. J., Hahn, P., Brown, Leudi, M., Shepherd, R. J., and Messing, J. 1981. The complete nucleotide sequence of an infectious clone of cauliflower mosaic virus by $\mathrm{m} 13 \mathrm{mp} 7$ shotgun sequencing. Nucl. Acids Res. 9:2871-2888.

Geri, C., Cecchini, E., Giannakou, M. E., Covey, S. N., and Milner, J. J. 1999. Altered patterns of gene expression in Arabidopsis elicited by cauliflower mosaic virus (CaMV) infection and by a CaMV gene VI transgene. Mol. Plant-Microbe Interact. 12:377-384.

Goldberg, K. B., Kiernan, J., and Shepherd, R. J. 1991. A disease syndrome associated with expression of gene VI of caulimoviruses may be a nonhost reaction. Mol. Plant-Microbe Interact. 4:182-189.

Gowda, S., Wu, F. C., Scholthof, H. B., and Shepherd, R. J. 1989. Gene VI of figwort mosaic virus (caulimovirus group) functions in posttranscriptional expression of genes on the full-length RNA transcript. Proc. Natl. Acad. Sci. U.S.A. 86:9203-9207.

Greene, A. E., and Allison, R. F. 1996. Depletion in the 3' untranslated region of cowpea chlorotic mottle virus transgene reduce recovery of recombinant viruses in transgenic plants. Virology 225:231-234.

Grimsley, N., Hohn, B., Hohn, T., and Walden, R. 1986. Agroinfection: An alternative route for viral infection of plants by using the Ti plasmid. Proc. Natl. Acad. Sci. U.S.A. 83:3282-3286.

Higuchi, R. 1990. Recombinant PCR. Pages 177-183 in: PCR Protocols: A Guide to Methods and Applications. M. A. Innes, D. H. Gelfand, J. J. Sninsky, and T. J. White, eds. Academic Press, Inc. San Diego, CA, U.S.A.

Hull, R. 2002. Matthews' Plant Virology. Academic Press, San Diego, CA, U.S.A.

Hull, R., and Covey, S. N. 1983. Does cauliflower mosaic virus replicate reverse transcription? Trends Biochem. Sci. 8:119-121.

Király, L., Bourque, J. and Schoelz, J. E. 1998. Temporal and spatial appearance of recombinant viruses formed between Cauliflower mosaic virus (CaMV) and CaMV Sequences present in transgenic Nicotiana bigelovii. Mol. Plant-Microbe Interact. 11:309-316.

Király, L., Cole, A. B., Bourque, J. E., and Schoelz, J E. 1999. Systemic cell death is elicited by the interaction of a single gene in Nicotiana clevelandii and gene VI of cauliflower mosaic virus. Mol. PlantMicrobe Interact. 12:919-925.

Kobayashi, K., Tsuge, S., Nakayashiki, H., Mise, K., Furusawa, I. 1998. Requirement of cauliflower mosaic virus open reading frame VI product for viral gene expression and multiplication in turnip protoplasts. Microbiol. Immunol. 42:377-386.

Laemmli, U. K. 1970. Cleavage of structural proteins during the assembly of head of bacteriophage T4. Nature 227:680-685.

Lawton, M. A., Tierney, M. A., Nakamura, I., Anderson, E., Komeda, Y., Dube, P., Hoffman, N., Fraley, R. T., and Beachy, R. N. 1987. Expression of a soybean B-conglycinin gene under the control of the cauli- flower mosaic virus $35 \mathrm{~S}$ and $19 \mathrm{~S}$ promoters in transformed petunia tissues. Plant Mol. Biol. 9:315-324.

Leisner, S. M., and Howell, S. H. 1992. Symptom variation in different Arabidopsis thaliana ecotypes produced by cauliflower mosaic virus. Phytopathology 82:1042-1046.

Maniatis, T., Fritsch, E. F., and Sambrook, J. 1982. Page 545 in: Molecular Cloning: A Laboratory Manual. Cold Spring Harbor Laboratory Press, Cold Spring Harbor, NY, U.S.A.

Melcher, U. 1989. Symptoms of cauliflower mosaic virus infection in Arabidopsis thaliana and turnip. Bot. Gaz. 150:139-147.

Moore, C. J., Sutherland, P. W., Forster, R. L. S., Gardner, R. C., and MacDiarmid, R. M. 2001. Dark green islands in plant virus infection are the result of posttranscriptional gene silencing. Mol. Plant-Microbe Interact. 14:939-946.

Murashige, T., and Skoog, F. 1962. A revised medium for rapid growth and bio-assays with tobacco tissue cultures. Physiol. Plant. 15:473-479.

Odell, J. T., and Howell, S. H. 1980. The identification, mapping and characterization of messenger RNA for $\mathrm{p} 66$, a cauliflower mosaic virus coded protein. Virology 102:349-359.

Palanichelvam, K., Cole, A. B., Shababi, M., and Schoelz, J. E. 2000. Agroinfiltration of Cauliflower mosaic virus gene VI elicits hypersensitive response in Nicotiana species. Mol. Plant-Microbe Interact. 13:1275-1279.

Palanichelvam, K., and Schoelz, J. E. 2002. A comparative analysis of the avirulence and translational transactivator functions of gene VI of cauliflower mosaic virus. Virology 293:225-233.

Park, H.-S., Himmelbach, A., Browning, K. S., Hohn, T., and Ryabova, L. A. 2001. A plant viral "reinitiation factor interacts with the host translational machinery. Cell 106:723-733.

Schardl, C. L., Byrd, A. D., Benzion, G., Altschuler, M. A., Hildebrand D. F., and Hunt, A. G. 1987. Design and construction of a versatile system for the expression of foreign genes in plants. Gene 61:1-11.

Schoelz, J. E., and Wintermantel, W. M. 1993. Expansion of viral host range through complementation and recombination in transgenic plants. Plant Cell 5:1669-1679.

Schoelz, J. E., Goldberg, K. B., and Kiernan, J. M. 1991. Expression of cauliflower mosaic virus (CaMV) gene VI in transgenic Nicotiana bigelovii complements a strain of CaMV defective in long-distance movement in nontransformed $N$. bigelovii. Mol. Plant-Microbe Interact. 4:350-355.

Scholthof, H. B., Wu, F. C., Gowda, S., and Shepherd, R. J. 1992. Regulation of cauliflower mosaic virus gene expression and the involvement of cis-acting elements on both viral transcripts. Virology 190:403-412.

Shintaku, M. H., Zhang, L., and Palukaitis, P. 1992. A single amino acid substitution in the coat protein of cucumber mosaic virus induces chlorosis in tobacco. Plant Cell 4:751-757.

Stratford, R., and Covey, S. N. 1989. Segregation of cauliflower mosaic virus symptom genetic determinants. Virology 172:451-459.

Takahashi, H., Shimamoto, K., and Ehara Y. 1989. Cauliflower mosaic virus gene VI causes growth suppression, development of necrotic spots and expression of defense-related genes in transgenic tobacco plants. Mol. Gen. Genet. 216:188-194.

Wintermantel, W. M., Anderson, E. J., and Schoelz, J. E. 1993. Identification of domains within gene VI of cauliflower mosaic virus that influence systemic infection of Nicotiana bigelovii in a light-dependent manner. Virology 196:789-798.

Wintermantel, W. M., and Schoelz, J. E. 1996. Isolation of recombinant viruses between cauliflower mosaic virus and a viral gene in transgenic plants under conditions of moderate selection pressure. Virology 223:156-164.

Xie, Z., Fan, B., Chen, C. and Chen, Z. 2001. An important role of an inducible RNA-dependent RNA polymerase in plant antiviral defense. Proc. Natl. Acad. Sci. U.S.A. 98:6516-6521.

Zijlstra, C., and Hohn, T. 1992. Cauliflower mosaic virus gene VI controls translation from dicistronic expression units in transgenic Arabidopsis plants. Plant Cell 4:1471-1484. 